PROCEEDINGS OF THE

AMERICAN MATHEMATICAL SOCIETY

Volume 128, Number 8, Pages 2465-2471

S 0002-9939(00)05734-8

Article electronically published on March 29, 2000

\title{
CHARACTERISTIC CLASSES FOR COMPLEX BUNDLES WITH TRIVIAL REAL REDUCTION
}

\author{
DUAN HAIBAO
}

(Communicated by Ralph Cohen)

\begin{abstract}
This note concerns itself with a theory of characteristic classes for those complex bundles whose real reductions are trivial.
\end{abstract}

\section{1}

For a topological space $X$ let $A_{n}(X)$ be the set of the complex $n$-bundles over $X$ obtained by furnishing the trivial real bundle $X \times R^{2 n} \rightarrow X$ a complex structure. Two bundles $\xi_{0}, \xi_{1} \in A_{n}(X)$ are considered to be equivalent if there exists some $\xi \in A_{n}(X \times I)$ so that $\xi \mid X \times i=\xi_{i}, i=0,1$. Denote by $B_{n}(X)$ the set of all such equivalence classes.

It should be noted that our partition on $A_{n}(X)$ by the equivalence classes is subtler than the one given by isomorphism classes of complex bundles.

2

Elements of $B_{n}(X)$ can be classified within homotopy theory. Let $C S_{n}$ be the space of all complex structures $J$ 's on the $2 n$-dimensional Euclidean space $R^{2 n}$. The operator

$$
K: C S_{n} \times R^{2 n} \rightarrow C S_{n} \times R^{2 n}
$$

defined by $K(J, v)=(J, J v)$ equips the trivial real vector bundle $C S_{n} \times R^{2 n} \rightarrow C S_{n}$ a complex structure. Denote by $\gamma_{n}$ the resulting complex $n$-bundle over $C S_{n}$. It will be called the canonical bundle over $C S_{n}$.

For two topological spaces $X, Y$, let $[X, Y]$ be the set of homotopy classes of continuous maps $X \rightarrow Y$.

Proposition 1. For any topological space $X$ the correspondence $h:\left[X, C S_{n}\right] \rightarrow$ $B_{n}(X), h([f])=f^{*} \gamma_{n}$, is a bijection.

Indeed the inverse of $h$ is seen as follows. For a $\xi \in B_{n}(X)$ consider the complex structure on $\xi$ as an $R$-linear morphism $J_{\xi}: X \times R^{2 n} \rightarrow X \times R^{2 n}$ of the real reduction of $\xi$. The map $f_{\xi}: X \rightarrow C S_{n}$ assigning to each $x \in X$ the complex structure $J_{\xi} \mid x \times R^{2 n} \in C S_{n}$ is continuous and satisfies $f_{\xi}^{*} \gamma_{n}=\xi$. It will be called the classifying map of $\xi$.

Received by the editors September 11, 1997.

2000 Mathematics Subject Classification. Primary 55R40; Secondary 55P62.

Key words and phrases. Vector bundles, characteristic classes, cohomology and rational homotopy theory.

This work was supported by NSFC Project 1977001.

(C)2000 American Mathematical Society 
The space $C S_{n}$ has two connected components, both diffeomorphic to the homogeneous space $S O(2 n) / U(n)$. Denote by $C S_{n}^{+}$the component that contains

$$
J_{0}=\left(\begin{array}{cc}
0 & 1 \\
-1 & 0
\end{array}\right) \oplus \cdots \oplus\left(\begin{array}{cc}
0 & 1 \\
-1 & 0
\end{array}\right)(n \text { copies })
$$

and by $C S_{n}^{-}$the other.

Let $1+c_{1}+\cdots+c_{n}$ be the total Chern characteristic class for the restricted bundle $\gamma_{n} \mid C S_{n}^{+}$. We describe $H^{*}\left(C S_{n}^{+} ; Z\right)$, the integral cohomology algebra of $C S_{n}^{+}$, in

Proposition 2. The classes $c_{i}, i=1, \cdots, n-1$, are all divisible by 2 . Further if we put $e_{i}=\frac{1}{2} c_{i}$, then $e_{1}, \cdots, e_{n-1}$ form a simple system of generators for $H^{*}\left(C S_{n}^{+} ; Z\right)$ [5, p.372] and are subject to the relations

$$
R_{i}: e_{i}^{2}-2 e_{i-1} e_{i+1}+2 e_{i-2} e_{i+2}-\cdots+(-1)^{i} e_{2 i}=0, \quad i=1, \cdots, n-1,
$$

with the convention $e_{k}=0, k>n-1$, being understood.

The cohomology algebra of the space $C S_{n}^{+}$has been determined for $Z_{2}$ coefficients by C. Miller [3], and for $Z_{p}$ ( $p$ a prime) coefficients by A. Borel [1. The proof of Proposition 2 will be postponed until the final Section 7 .

By the first $\left[\frac{n+1}{2}\right]-1$ relations each $e_{2 i}$ can be expressed as a polynomial $g_{i}$ in $e_{\text {odd }}$. For instance the first four such polynomials are given by

$$
\begin{aligned}
& g_{1}=e_{1}^{2}, \\
& g_{2}=2 e_{1} e_{3}-e_{1}^{4}, \\
& g_{3}=e_{3}^{2}+2 e_{1} e_{5}-4 e_{1}^{3} e_{3}+2 e_{1}^{6}, \\
& g_{4}=2 e_{3} e_{5}+2 e_{1} e_{7}-6 e_{1}^{2} e_{3}^{2}+8 e_{1}^{5} e_{3}-3 e_{1}^{8}-4 e_{1}^{3} e_{5} .
\end{aligned}
$$

Consequently substituting $e_{2 i}$ by $g_{i}$ in the remaining $n-\left[\frac{n+1}{2}\right]$ relations gives rise to equations

$$
h_{k}=0, k=\left[\frac{n+1}{2}\right], \cdots, n-1
$$

where each $h_{k}$ is a polynomial in $e_{\text {odd }}$ of degree $4 k$. For example when $n=9$ these polynomials are

$$
\begin{aligned}
& h_{5}=e_{5}^{2}-2 g_{2} g_{3}+2 e_{3} e_{7}-2 g_{1} g_{4}, \\
& h_{6}=g_{3}^{2}-2 e_{5} e_{7}+2 g_{2} g_{4}, \\
& h_{7}=e_{7}^{2}-2 g_{3} g_{4}, \\
& h_{8}=g_{4}^{2} .
\end{aligned}
$$

Consider the algebra

$$
\Phi=\left\{\begin{array}{l}
Z\left[d_{1}, d_{3}, \cdots, d_{n-2}\right] \otimes \Lambda_{Z}\left(v_{2 n+1}, v_{2 n+5}, \cdots, v_{4 n-5}\right) \text { when } n \text { is odd } \\
Z\left[d_{1}, d_{3}, \cdots, d_{n-1}\right] \otimes \Lambda_{Z}\left(v_{2 n-1}, v_{2 n+3}, \cdots, v_{4 n-5}\right) \text { when } n \text { is even }
\end{array}\right.
$$

the tensor product of the polynomial algebra in $d_{o d d}$ with the exterior algebra in $v_{j}$. It is graded by

$$
\operatorname{dim}\left(d_{i}\right)=2 i \text { and } \operatorname{dim}\left(v_{j}\right)=j .
$$

The differential $\delta: \Phi \rightarrow \Phi$ of degree 1 given by

$$
\delta\left(d_{i}\right)=0, \delta\left(v_{j}\right)=h_{\left[\frac{j+1}{4}\right]}\left(d_{1}, d_{3}, \cdots\right)
$$


furnishes the algebra $\Phi$ with the structure of a differential graded commutative free algebra over $Z$. Moreover since $C S_{n}^{+}$is a symmetric space, Proposition 2 implies

Proposition 3. The algebra map $l:(\Phi, \delta) \rightarrow\left(H^{*}\left(C S_{n}^{+} ; Z\right), \delta=0\right)$ defined by $l\left(d_{i}\right)=e_{i}, l\left(v_{j}\right)=0$ is the minimal model (over $Z$ ) for the space $C S_{n}^{+}$(cf. [2] p.158]).

The connected component decomposition $C S_{n}=C S_{n}^{+} \sqcup C S_{n}^{-}$yields a natural partition $\left[X ; C S_{n}\right]=\left[X, C S_{n}^{+}\right] \sqcup\left[X, C S_{n}^{-}\right]$. Let $B_{n}^{ \pm}(X)$ be the $h$-image of $\left[X ; C S_{n}^{ \pm}\right]$ in $B_{n}(X)$. Since $C S_{n}^{+}$and $C S_{n}^{-}$are mutually diffeomorphic, a theory of characteristic classes on $B_{n}^{+}(X)$ corresponds to one on $B_{n}^{-}(X)$. So our remaining discussion will focus on the former.

From now on assume that our space $X$ is either a simply connected cell complex or has the rational homotopy type as a product of odd dimensional spheres. Let $\left(\Phi(X), \delta_{X}\right)$ be the rational minimal model of $X$. For a continuous map $f: X \rightarrow$ $C S_{n}^{+}$, let $\Phi(f)$ be the homotopy class of a minimal model $\Phi \otimes Q \rightarrow \Phi(X)$ of $f$. Denote by $[\Phi \otimes Q, \Phi(X)]$ the set of homotopy classes of differential graded algebra maps $\Phi \otimes Q \rightarrow \Phi(X)$. Consider the functorial correspondence

$$
\left[X, C S_{n}^{+}\right] \rightarrow[\Phi \otimes Q, \Phi(X)]
$$

given by $[f] \rightarrow \Phi(f)$ [2, p.173].

Definition. Let $f: X \rightarrow C S_{n}^{+}$be the classifying map of a $\xi \in B_{n}^{+}(X)$. The sets

$$
d_{i}(\xi)=\left\{g\left(d_{i} \otimes 1\right) \in \Phi(X) \mid g \in \Phi(f)\right\}
$$

will be called the primary Chern characteristic sets of $\xi$. The sets

$$
v_{j}(\xi)=\left\{g\left(v_{j} \otimes 1\right) \in \Phi(X) \mid g \in \Phi(f)\right\}
$$

will be called the secondary Chern characteristic sets of $\xi$.

Since the forms $d_{i}$ are closed in $\Phi$, each set $d_{i}(\xi)$ consists of closed forms and the difference of any two such forms is a coboundary. Therefore passing to cohomology yields an unique class $\left\{d_{i}(\xi)\right\} \in H^{2 i}(X ; Q)$. The following corollary of Propositions 2 and 3 implies that these classes constitute nothing essentially new

Proposition 4. In the rational cohomology $H^{*}(X ; Q)$ the usual Chern characteristic classes $c_{1}(\xi), \cdots, c_{n}(\xi)$ of a $\xi \in B_{n}^{+}(X)$ can be given in terms of the primary Chern characteristic sets by the formulas

$$
c_{2 k+1}(\xi)=2\left\{d_{2 k+1}(\xi)\right\} ; c_{2 k}(\xi)=2 g_{k}\left(\left\{d_{1}(\xi)\right\},\left\{d_{3}(\xi)\right\}, \cdots\right) ; c_{n}(\xi)=0 .
$$

5

It is the secondary Chern characteristic sets $v_{j}(\xi)$ that are of our interest. We observe that some homotopy and cohomology invariants can be extracted from the sets $v_{j}(\xi)$ even though the forms $v_{j} \otimes 1$ themselves are not closed in $\Phi \otimes Q$.

Observation 1. By rational homotopy theory the forms $v_{j} \otimes 1$ constitute a basis for $\operatorname{Hom}\left(\pi_{\text {odd }}\left(C S_{n}^{+}\right), Q\right)$ and for a continuous map $f: X \rightarrow C S_{n}^{+}$, the set of induced chain maps

$$
\Phi(f): \Phi \otimes Q \rightarrow \Phi(X)
$$


module decompositables, agrees with the dual action of the induced homotopy homomorphism

$$
f_{*}: \pi_{*}(X) \rightarrow \pi_{*}\left(C S_{n}^{+}\right) .
$$

(See [2, p.175], or Lemma 3 in Section 6.) Thus the element

$$
\pi_{j}(\xi)=v_{j}(\xi) \text { module decompositables }
$$

is well defined in $\operatorname{Hom}\left(\pi_{\text {odd }}(X) ; Q\right)=\pi_{\text {odd }}(X) \otimes Q$.

Observation 2. Assume that our space $X$ has the rational homotopy type as a product of odd-dimensional spheres. Then the minimal model $\Phi(X)$ agrees with the rational cohomology of $X$. Thus each set $v_{j}(\xi)$ actually consists of elements in $H^{*}(X ; Q)$.

Observation 3. Suppose the bundle $\xi \in B_{n}(X)$ with classifying map $f_{\xi}$ is such that

$$
d_{i}(\xi)=\{0\}, i=1,3, \cdots
$$

(this happens, in particular, when $X$ is $2 n-2$ connected). Let $\delta_{X}$ be the differential of $\Phi(X)$. Then

$$
\begin{aligned}
\delta_{X} \Phi\left(f_{\xi}\right)\left(v_{j}\right) & =\Phi\left(f_{\xi}\right) \delta v_{j}=\Phi\left(f_{\xi}\right) h_{\frac{j+1}{4}}\left(d_{1}, d_{3}, \cdots\right) \\
& =h_{\frac{j+1}{4}}\left(d_{1}(\xi), d_{3}(\xi), \cdots\right)=0
\end{aligned}
$$

indicates that $v_{j}(\xi)$ consists of closed forms. Similarly one can show that the difference of any two forms in $v_{j}(\xi)$ is a coboundary. That is, each set $v_{j}(\xi)$ survives to a unique element of $H^{*}(X ; Q)$.

The homotopy and cohomology invariants associated with a $\xi \in B_{n}^{+}(X)$ in the previous section can well be nontrivial even if the usual Chern classes $c_{1}(\xi)$, $\cdots, c_{n-1}(\xi)$ all vanish.

Let $S O(2 n)$ be the special orthogonal group of order $2 n$, and let $f_{k}: S O(2 n) \rightarrow$ $C S_{n}^{+}$, for an integer $k \in Z$, be defined by $f_{k}(g)=g^{k} J_{0} g^{\prime k}$, where $J_{0} \in C S_{n}^{+}$is specified in Section 3 and ' is the transpose operator. Denote by $\xi_{k}$ the induced bundle $f_{k}^{*} \gamma_{n}$.

Theorem. For the sequence of complex n-bundles $\left\{\xi_{k} \in B_{n}^{+}(S O(2 n)) \mid k \in Z\right\}$

1) the secondary Chern characteristic classes $\pi_{i}$ and $v_{i}$ are distinctive;

2) the usual Chern characteristic classes $c_{i}$ vanish.

Using the grading $\bigoplus \Phi^{r}(X)$ of the model $\Phi(X)$ a graded vector space $I(X)=$ $\bigoplus I^{r}(X)$ can be introduced by setting

$$
I^{r}(X)=\Phi^{r}(X) / \text { decompositable forms. }
$$

For instance it follows from Proposition 3 that

\section{Lemma 1.}

$$
I\left(C S_{n}^{+}\right)=\left\{\begin{array}{c}
\operatorname{span}_{Q}\left\{e_{1}, e_{3}, \cdots, e_{n-1}, v_{2 n-1}, v_{2 n+3}, \cdots, v_{4 n-5}\right\} \text { if } n \text { is even, } \\
\operatorname{span}_{Q}\left\{e_{1}, e_{3}, \cdots, e_{n-2}, v_{2 n+1}, v_{2 n+5}, \cdots, v_{4 n-5}\right\} \text { if } n \text { is odd. }
\end{array}\right.
$$


For a compact connected Lie group $G$ the minimal model $\Phi(G)$ agrees with $H^{*}(G ; Q)$. From the well known isomorphisms

$$
\begin{aligned}
& H^{*}(U(n) ; Q)=\Lambda_{Q}\left(y_{1}, y_{3}, \cdots, y_{2 n-1}\right), \\
& H^{*}(S O(2 n) ; Q)=\Lambda_{Q}\left(x_{5}, x_{7}, \cdots, x_{4 n-5}, w_{2 n-1}\right)
\end{aligned}
$$

(where the generators $y_{i}, x_{i}$ and $w_{2 n-1}$ are primitive with the suffixes indicating their dimensions) we get

\section{Lemma 2.}

$$
\begin{aligned}
& I(U(n))=\operatorname{span}_{Q}\left\{y_{1}, y_{3}, \cdots, y_{2 n-1}\right\}, \\
& I(S O(2 n))=\operatorname{span}_{Q}\left\{x_{3}, x_{7}, \cdots, x_{4 n-5}, w_{2 n-1}\right\} .
\end{aligned}
$$

A continuous map $f: X \rightarrow Y$ induces a well defined homomorphism $I(f)$ : $I(Y) \rightarrow I(X)$. We recall from [2, p.175] that

Lemma 3. There is a canonical isomorphism $I^{r}(X) \rightarrow \operatorname{Hom}\left(\pi_{r}(X), Q\right)$ which is natural with respect to homomorphisms induced by maps $X \rightarrow Y$.

For an integer $k \in Z$ let $l_{k}$ be the self-map of $S O(2 n)$ defined by $g \rightarrow g^{k}$. Since the induced algebra map $H^{*}(S O(2 n) ; Q) \rightarrow H^{*}(S O(2 n) ; Q)$ is given by $l_{k}^{*}\left(x_{i}\right)=$ $k x_{i}, l_{k}^{*}\left(w_{2 n-1}\right)=k w_{2 n-1}$, we have

Lemma 4. The induced endomorphism $I\left(l_{k}\right)$ of $I(S O(2 n))$ is multiplication by $k$.

Clearly the map $f_{1}: S O(2 n) \rightarrow C S_{n}^{+}$is the projection of the standard fibration

$$
U(n) \subset S O(2 n) \rightarrow C S_{n}^{+} .
$$

Applying the natural transformation $\operatorname{Hom}(; Q)$ to the homotopy exact sequence of $f_{1}$ yields the exact sequence of vector spaces

$$
\cdots \leftarrow I^{r}(U(n)) \leftarrow I^{r}(S O(2 n)) \stackrel{I^{r}\left(f_{1}\right)}{\longleftarrow} I^{r}\left(C S_{n}^{+}\right) \leftarrow I^{r-1}(U(n)) \leftarrow \cdots .
$$

A dimension comparison discussion based on Lemmas 1 and 2 concludes

Lemma 5. The homomorphism $I^{r}\left(f_{1}\right): I^{r}\left(C S_{n}^{+}\right) \rightarrow I^{r}(S O(2 n))$ is

1) an isomorphism for $r=2 n+1,2 n+5, \cdots, 4 n-5$ when $n$ is odd, and for $r=2 n+3,2 n+7, \cdots, 4 n-5$ when $n$ is even;

2) an injection for $r=2 n-1$ when $n$ is even.

Proof of the Theorem. Since $\left.f_{k}=f_{1} \circ l_{k}, 1\right)$ is immediate from Lemmas 4 and 5 .

For 2) consider the flag manifold $S O(2 n) / T^{n}$ as the set of orthogonal decompositions of $R^{2 n}$ into oriented 2-planes $R^{2 n}=L_{1} \oplus \cdots \oplus L_{n}$. Assigning to each $L_{1} \oplus \cdots \oplus L_{n} \in S O(2 n) / T^{n}$ with $\omega_{1} \oplus \cdots \oplus \omega_{n} \in C S_{n}^{+}$, where $\omega_{i}$ is the $\frac{\pi}{2}$ rotation on $L_{i}$ with respect to the orientation, yields a map $g: S O(2 n) / T^{n} \rightarrow C S_{n}^{+}$. In fact $f_{1}$ factors through the space $S O(2 n) / T^{n}$ in the fashion

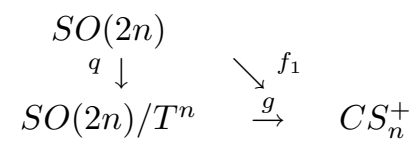

where $q$ is the standard projection (for $T^{n} \subset U(n)$ ). Since the induced bundle $g^{*} \gamma_{n}$ admits a canonical splitting into complex line bundles (this is indicated by 
our description of $g$ ) we find

$$
\begin{aligned}
g^{*} c_{r}\left(\gamma_{n}\right)= & \text { the } r \text { th elementary symmetric polynomial } \\
& \text { in some } x_{1}, \cdots, x_{n} \in H^{2}\left(S O(2 n) / T^{n} ; Q\right) .
\end{aligned}
$$

The proof of 2) is now completed by the facts $H^{2}(S O(2 n) ; Q)=0$ and $f_{k}=f_{1} \circ l_{k}=$ $g \circ q \circ l_{k}$.

It also follows from Lemmas 2 and 5 that a sequence of complex $n$-bundles over each of the spheres

$$
S^{r}, r=\left\{\begin{array}{c}
2 n+1,2 n+5, \cdots, 4 n-5 \text { when } n \text { is odd, } \\
2 n-1,2 n+3, \cdots, 4 n-5 \text { when } n \text { is even, }
\end{array}\right.
$$

with distinctive secondary Chern classes $v_{r} \in H^{r}\left(S^{r} ; Q\right)=Q$ (in the sense of Observation 3) can be obtained from the bundles $\xi_{k}, k \in Z$.

This section is devoted to a proof of Proposition 2.

Let $s_{1}, \cdots, s_{2 n}$ be the standard vector space basis for $R^{2 n}$, and let $S^{2 n-2}$ be the unit sphere in the subspace $R^{2 n-1}$ spanned by $s_{1}, \cdots, s_{2 n-1}$. The map

$$
\pi: C S_{n}^{+} \rightarrow S^{2 n-2}, \quad \pi(J)=J s_{2 n} \in S^{2 n-2}
$$

is a fiber bundle projection whose fiber inclusion over $s_{2 n-1} \in S^{2 n-2}$, with respect to the standard splitting $R^{2 n}=\operatorname{span}\left\{s_{1}, \cdots, s_{2 n-2}\right\} \oplus \operatorname{span}\left\{s_{2 n-1}, s_{2 n}\right\}$, is

$$
i_{n}: C S_{n-1}^{+} \rightarrow C S_{n}^{+}, \quad i_{n}\left(J^{\prime}\right)=J^{\prime} \oplus\left(\begin{array}{cc}
0 & 1 \\
-1 & 0
\end{array}\right) .
$$

The proof proceeds by an induction on $n$. Let $\gamma$ be the Hopf line bundle over the 2-sphere $S^{2}$. Since $C S_{2}^{+}=S^{2}$ and since $\gamma_{2}=\gamma \oplus \gamma$, Proposition 2 is true for $n=2$. Assume that it has been proved for $n-1$.

Let $c_{r}\left(\gamma_{n}\right)$ be the $r$ th Chern class of $\gamma_{n}$. Since $H^{*}\left(C S_{n}^{+} ; Z\right)$ is torsion free and since the real reduction of $\gamma_{n}$ is trivial, the 2-divisibility of $c_{r}\left(\gamma_{n}\right)$ follows from $c_{r}\left(\gamma_{n}\right) \equiv 0 \bmod 2$.

Consider $e_{r}\left(\gamma_{n}\right)=\frac{1}{2} c_{r}\left(\gamma_{n}\right), r=1,2, \cdots, n-1$. By the naturality of Chern classes with respect to induced bundles we get from $i_{n}^{*} \gamma_{n}=\gamma_{n-1} \oplus \epsilon$ that

1) $i_{n}^{*}\left(e_{r}\left(\gamma_{n}\right)\right)=e_{r}\left(\gamma_{n-1}\right), r=1,2, \cdots, n-2$,

2) $i_{n}^{*}\left(e_{n-1}\left(\gamma_{n}\right)\right)=0$ where $i_{n}^{*}: H^{*}\left(C S_{n}^{+} ; Z\right) \rightarrow H^{*}\left(C S_{n-1}^{+} ; Z\right)$ is the induced homomorphism. Combined with the inductive hypothesis 1$)$ indicates

Lemma 6. The bundle $\pi$ has Leray-Hirsch property [5, p.365].

It now follows from 2$)$ that, if we let $a \in H^{2 n-2}\left(S^{2 n-2} ; Z\right)$ be a generator, then 3) $e_{n-1}\left(\gamma_{n}\right)=k \pi^{*} a$ for some $k \in Z$.

We evaluate the integer $k$ in

Lemma 7. $k= \pm 1$.

Proof. Let $\tau$ be the tangent bundle of the base $S^{2 n-2}$. It has Euler class $\pm 2 a$. The induced bundle $\pi^{*} \tau$ is a subbundle of $\gamma_{n}$ whose orthogonal complement $\left(\pi^{*} \tau\right)^{\perp}$ over a $J \in C S_{n}^{+}$is the 2-plane spanned by $s_{2 n}, J s_{2 n} \in R^{2 n}$. Thus $\gamma_{n}$ has a ready made 
decomposition into the Whitney sum of complex bundles $\pi^{*} \tau \oplus\left(\pi^{*} \tau\right)^{\perp}$ in which the second summand is trivial. This implies

$$
c_{n-1}\left(\gamma_{n}\right)=c_{n-1}\left(\pi^{*} \tau\right) \text {. }
$$

However the top Chern class $c_{n-1}\left(\pi^{*} \tau\right)$ can be recognized as the Euler class of $\pi^{*} \tau$, which is $\pm 2 \pi^{*} a$.

The first statement of Proposition 2 has now been proved by Lemmas 6 and 7 (as well as our inductive hypothesis).

Finally since the real reduction of $\gamma_{n}$ is trivial, the formulas expressing the Pontrjagin classes of $\gamma_{n}$ in terms of its Chern classes [4, p.177] give rise to the equations

$$
c_{r}\left(\gamma_{n}\right)^{2}-2 c_{r-1}\left(\gamma_{n}\right) c_{r+1}\left(\gamma_{n}\right)+-\cdots \pm 2 c_{1}\left(\gamma_{n}\right) c_{2 r-1}\left(\gamma_{n}\right) \mp 2 c_{2 r}\left(\gamma_{n}\right)=0 .
$$

Dividing both sides by the common divisor 4 yields the relations $R_{1}-R_{n-1}$.

\section{REFERENCES}

[1] A. Borel, Sur la cohomologie des espaces fibres principaux et des espaces homogenes de groupes de Lie compacts, Annals of Math., Vol. 57(1953), 115-207. MR 14:490e

[2] P.A. Griffiths and J.W. Morgan, Rational homotopy theory and differential forms, Birkhauser Boston, 1981. MR 82m:55014

[3] C.E. Miller, The topology of rotation groups, Annals of Math., Vol. 57(1953), 90-114. MR 14:673b

[4] J.W. Milnor and J.D. Stasheff, Characteristic classes, Ann. Math. Studies, No. 76, Princeton University Press, 1974. MR 55:13428

[5] R.M. Switzer, Algebraic topology-homotopy and homology, Springer-Verlag Berlin-Heidelberg, 1975. MR 52:6695

Department of Mathematics, Peking University, Beijing 100871, People's Republic of CHINA

E-mail address: dhb@sxx0.math.pku.edu.cn 